\title{
QB: Embracing the future of quantitative understanding and engineering of life
}

\author{
Xuegong Zhang ${ }^{1,2,3, *}$ \\ ${ }^{1}$ Bioinformatics Division, BNRist and Department of Automation, Tsinghua University, Beijing 100084, China \\ 2 MOE Key Lab of Bioinformatics and School of Life Sciences, Tsinghua University, Beijing 100084, China \\ ${ }^{3}$ Center for Synthetic \& Systems Biology, Tsinghua Uniersity, Beijing 100084, China \\ * Correspondence: zhangxg@tsinghua.edu.cn
}

As a new inter- and multi-disciplinary forum for modeling, engineering and understanding life, our journal Quantitative Biology $(Q B)$ is celebrating its 6th anniversary and the start of a new Editorial Board in this issue. The past 6 years have evidenced tremendous progress in the research on computational biology, systems biology and synthetic biology. Omics studies have expanded from genomes and transcriptomes to multiple aspects of epigenomes and their interactions. People's understanding and modeling on genomes is becoming $3 \mathrm{D}$ or $4 \mathrm{D}$. The development of high throughput single-cell genomics technologies has aroused huge enthusiasm for building the Human Cell Atlas as an ultimate reference for all future studies on human. And the rapid popularity of sequencing technologies in parallel with big-data technologies resulted in large-scale disease study projects like the UK BioBank project, the US All-of-Us Research Program, and the China Precision Medicine plan, all based on the collection of massive population health and genomics data. In the broad field of synthetic biology, technologies are enabling not only the "editing" and "writing" of genomic segments, but also the synthesis of entire eukaryote chromosomes. On the more basic level, scientists are beginning to design and make proteins that do not exist in nature, and even trying to expand the genetic alphabet by devising "unnatural" nucleotide bases and adding those new letters into genomes of living organisms like Escherichia coli. Such advances in basic research are accompanied with enthusiastic development of biological technologies for diagnosing and curing diseases, such as lipid biopsy diagnosis, immuno-therapies and stem-cell therapies for cancer and degenerative diseases. In the meanwhile, theoretical studies on modeling basic biological procedures like development, tumorigenesis and microevolution have also seen great advancement.

The broad and rapid development of all fields of quantitative biology is generating and accumulating huge amount of data. More and more large-scale biological studies are becoming data-driven. This trend coincides with the new booming of machine learning and artificial intelligence in information science and technology. Empowered with new models like deep neural networks and reconfigurable high-performance computing hardware, machine learning algorithms showed great potential for making sense out of huge amount of data. This potential is also being explored on many biological and medical studies. Machine learning methods were originally developed for enabling computers to perform on perceptive tasks that human beings or some animals are capable of. Scientists are now hoping that the technology can also be extended to discover hints or patterns about complicated biological systems from highly complex data that challenge the perception capability of naked human intelligence. With the guidance of theoretical biology principles and models, hybrid data- and model-driven efforts can be expected to accelerate our understanding of life. In the meanwhile, the deeper quantitative understanding of natural life at molecular, cellular and system levels will also promote the development of artificial systems, both computer systems and synthetic biological systems, that can mimic, assist or supplement some aspects of the function of natural life.

In the past 6 years, QB not only witnessed those exciting advancement toward quantitative understanding of life, but also made its own remarkable contributions as a forum that links many disciplines that would be 
scattered in multiple traditional journals. Thanks to the great work of the founding Editorial Board under the leadership of Drs. Michael Q. Zhang and Chao Tang, QB has already published $>150$ papers including comprehensive reviews from leading scientists and cutting-edge research articles from most active researchers. They spanned a broad spectrum of research topics including bioinformatics methods, tools and databases for NGS especially single-cell sequencing data; models and algorithms for analyzing biological interactions, regulations and networks; 3D genomes and epigenomic analyses; quantitative analysis of microbes and microbiomes; protein and RNA structures; theoretical and technological developments in synthetic biology; mathematical and theoretical modeling of biological molecules, systems and evolution; and applications of machine learning in biology and medicine, etc. They reflected latest progress and insightful perspectives in the field and have been well received by the community. The current issue adds to the list a special collection of papers that focus on quantitative and systems studies of pharmacology.

Along with the many new advancement in science and technology, scientific publication is also entering a new era as traditional publication models are being challenged by the current open-access trend. Thanks to the support of our sponsoring institutions Tsinghua University and Peking University, QB has been making great efforts to make open-access for all papers free of charge for both authors and readers while still keep the traditional publication on printed issues. We are also open to explore new sustainable models for publication that can better serve the community in the future. For details on the Journal, please check either: https://www. springer.com/40484 or http://journal.hep.com.cn/qb. The submission, review and editorial communications of QB all take place through the well-known ScholarONE system at https://mc.manuscriptcentral.com/qb. The newly expanded international Editorial Board aims to make $Q B$ an exciting better home for interdisciplinary quantitative biology-related research and communications. The handling and peer-reviewing process is among the quickest in the area. And we especially value submissions of innovational work that might be treated unfairly with prejudice by some other journals. Biology is a science with long history, but the quantitative understanding of life is still at its early phase. We hope QB will encourage and stimulate more creative models, revolutionary approaches and innovative technologies for understanding and engineering life systems at all levels.

Xuegong Zhang

Executive Editor-in-Chief 